\title{
Systemic inflammation is associated with depressive symptoms differentially by sex and race: a longitudinal study of urban adults
}

\author{
May A. Beydoun ${ }^{1} \cdot$ Hardeep K. Obhi ${ }^{1} \cdot$ Jordan Weiss $^{1,2} \cdot$ Jose A. Canas $^{3} \cdot$ Hind A. Beydoun ${ }^{4} \cdot$ Michele K. Evans $^{1} \cdot$ \\ Alan B. Zonderman ${ }^{1}$
}

Received: 30 August 2018 / Revised: 18 February 2019 / Accepted: 5 March 2019 / Published online: 24 April 2019

(c) The Author(s) 2019. This article is published with open access

\begin{abstract}
Systemic inflammation may influence trajectories of depressive symptoms over time, perhaps differentially by sex and race. Inflammatory markers and the Center for Epidemiologic Studies-Depression scale [total score: CES- $D_{\text {total }}$ and four distinctive domains: somatic complaints, depressed affect, positive affect and interpersonal problems] were examined among African-American (AA) and White urban adults participating in the Healthy Aging in Neighborhoods of Diversity across the Life Span (HANDLS) study [2004-2013, Age base $_{30}$ 30-64 y, mean \pm SD follow-up time: $4.64 \pm 0.93 \mathrm{y}, N=150$ (with cytokine data) to $N=1,767$ (with other inflammatory markers)]. Findings suggest that serum concentrations of highsensitivity C-reactive protein (hsCRP), z-inflammation composite score [ICS, combining elevated hsCRP and ESR with low serum albumin and iron], and serum interleukin (IL) $1 \beta$ were positively associated with $\Delta$ CES- $D_{\text {total }}(\Delta$ : annual rate of increase) among Whites only. IL-12 was directly related to $\triangle$ CES-D total $_{\text {among men and AA. The race-specific associations }}$

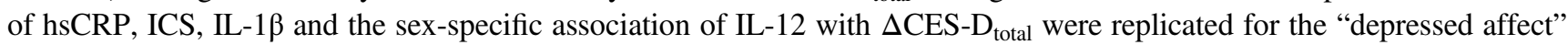
domain. Similarly, among men, lower serum albumin and higher ICS were linked with higher baseline "somatic complaints". IL-10 among AA and IL-12 among men were inversely related to $\Delta$ "positive affect", while "interpersonal problems" were cross-sectionally associated with IL-6 among AA and IL-10 among Whites. Finally, baseline ICS was positively associated with incident "elevated depressive symptoms" (EDS: CES-D total $\geq 16$ ) among AA (HR $=1.28,95 \%$ CI: $1.04-1.56, P=0.017)$. Overall, systemic inflammation was directly linked to increased depressive symptoms over time and at baseline, differentially across sex and race groups. More longitudinal research is needed to replicate our findings.
\end{abstract}

Co-senior authors: Michele K. Evans and Alan B. Zonderman

Supplementary information The online version of this article (https:// doi.org/10.1038/s41380-019-0408-2) contains supplementary material, which is available to authorized users.

May A. Beydoun

baydounm@mail.nih.gov

1 Laboratory of Epidemiology and Population Sciences, National Institute on Aging, NIA/NIH/IRP, Baltimore, MD, USA

2 Population Studies Center and the Leonard Davis Institute of Health Economics, University of Pennsylvania, Philadelphia, PA, USA

3 Johns Hopkins All Children's Hospital, St. Petersburg, FL, USA

4 Department of Research Programs, Fort Belvoir Community Hospital, Fort Belvoir, VA, USA

\section{Introduction}

Depression is characterized by altered mood and poor cognitive function $[1,2]$ and is associated with a proinflammatory process which can lead to increased morbidity from cardiovascular disease [3, 4]. A number of psychoneuroimmunological dysfunctional processes have been proposed to help elucidate the origins of depression [2]. Indeed, depressed adults are observed to have activated peripheral immune systems with exaggerated proinflammatory cytokine production and abnormalities in neurotransmitter metabolism, neuroendocrine functions and regional brain activity. These aberrant pathways could increase the number of depressive symptoms [2]. Acute cytokine administration to humans and animals triggers sickness behavior, which shares common features with depression [2]. In addition, chronic stress is associated with a proinflammatory phenotype among adults leading to recurrent depressive episodes [2]. 
The Center for Epidemiological Studies-Depression scale can be used to extract elevated depressive symptoms (EDS) and can quantify total and domain-specific (e.g., depressed affect $v s$. somatic complaints) depressive symptom scores in populations. Systemic inflammation can be quantified by increased proinflammatory cytokines (e.g., interleukins: IL$1 \beta$, IL-6, IL-12 and IL-18) vs. decreased anti-inflammatory cytokines (e.g. IL-10) as well as the high-sensitivity Creactive protein (hsCRP) levels, whose expression is triggered by IL-6 [1, 5, 6]. Inflammation is also accompanied by an increased level of fibrinogen (proxied by the Erythrocyte Sedimentation Rate, ESR) [7], as well as reductions in serum transferrin saturation (and/or serum iron) and in serum albumin [8]. Importantly, depression was linked to systemic inflammation in recent large epidemiological studies [3, 9-20]. Nevertheless, longitudinal relationships between systemic inflammatory markers and depressive symptoms in urban populations are under-studied, particularly when those relationships are examined across sex, race- and domains of depressive symptoms.

Using the longitudinal data on an ethnically diverse urban sample, our study has two objectives. First, we will assess the cross-sectional (i.e., baseline vs. baseline) and longitudinal (i.e., baseline vs. annual rate of change $(\Delta)$; baseline vs. incident EDS) relationships of systemic inflammation markers with depressive symptoms. We hypothesize that depressive symptoms are worsened by systemic inflammation. Second, we examine the crosssectional and longitudinal relationships of systemic inflammation with specific domains of depressive symptoms. All these associations will be tested separately by sex and race.

\section{Materials And Methods}

\section{Database}

The Healthy Aging in Neighborhoods of Diversity across the Life Span (HANDLS) study is an ongoing prospective cohort study initiated in 2004. HANDLS examines health disparities associated with race and socioeconomic status. Using an area probability design, the study recruited a representative sample of African-Americans (AA) and Whites with baseline ages 30-64 years residing in Baltimore, Maryland [21]. The baseline visit (visit 1) had two phases. Phase I conducted participant screening and recruitment and administered a general household interview. Phase II consisted of in-depth examinations in mobile Medical Research Vehicles (MRVs). Follow-up examinations were performed in the MRVs. Following access to a protocol booklet in layman's terms coupled with a video describing procedures and re-contacts, written informed consent was obtained. The National Institute on Environmental Health Sciences Institutional Review Board of the National Institutes of Health approved the study protocol.

Our current study extracted longitudinal data from baseline (visit 1, performed in 2004-2009) designated as visit 1 and first follow-up examination (visit 2, performed in 2009-2013, mean \pm SD follow-up interval $=4.65 \mathrm{y} \pm 0.93 \mathrm{y}$ ) designated as visit 2 .

\section{Study Sample}

The initial HANDLS sample consisted of 3,720 participants (Sample 1). Dietary data were available for $N=2,177$ baseline participants ( $24 \mathrm{~h}$ dietary recalls) while depressive symptoms data were available for $N=2,736$ and $N=$ 2,239 at visits 1 and 2 , respectively. Baseline systemic inflammatory marker data were complete among $N=2,580$ [serum high sensitivity C-reactive protein (hsCRP), Erythrocyte Sedimentation Rate (ESR), albumin and iron] and among $N=244-259$ for cytokines (interleukins, IL-1 $\beta$, IL6, IL-10, IL-12 and IL-18). For participants with complete dietary data, depressive symptoms were complete on 1 or 2 visits for $N=1,991$ subjects. Inclusion and exclusion criteria were determined based on completeness of outcomes, exposures and covariates, with no other sample restrictions. Excluding missing data on baseline exposures and covariates along with missing data on depressive symptoms at both visits yielded two final analytic samples [Sample 2A: $N_{2 A}=1,767$; repeated observations $N_{2 A}^{\prime}=3,054$ for data complete on serum hsCRP, ESR, albumin and iron; Sample 2B: $N_{2 B}=150$; repeated observations $N_{2 B}^{\prime}=260$ for data complete on all cytokines]. Participants in Sample 2A differed significantly from the remaining Sample 1 of HANDLS by age $(+0.72$ y older, $P=0.019)$, with a reduced proportion of AA (56.7 vs. 61.3\%, $P=0.005)$. Compared to Sample 1, Sample 2B had a higher proportion of AA (75.3 vs. 58.4\%, $P<0.001$ ), but lower proportions of men ( 35 vs. $46 \%, P=0.008$ ) and individuals above poverty (48 vs. 59\%, $P=0.006$ ). A two-stage Heckman selection model was used to adjust for potential selection bias associated with these sociodemographic factor differentials.

\section{Depressive symptoms}

Measures of depressive symptoms at each visit were available using the 20-item Center of Epidemiological Studies-Depression (CES-D), a self-reported symptom rating scale assessing affective and depressed mood [22], which has good psychometric properties in various studies of older adults [23]. A total CES-D (CES-D total $)$ score $\geq 16$ reflects elevated depressive symptoms (EDS) [24]

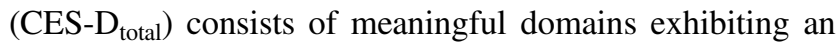
invariant factor structure between the National Health and 
Nutrition Examination Survey I and pilot HANDLS data [25]. Thus, our hypotheses were tested using the total score and domain-specific CES-D scores: (1) Somatic complaints; (2) Depressive affect; (3) Positive affect and (4) Interpersonal problems [25].

\section{Measures of systemic inflammation and composite score}

Blood obtained during the MRV baseline visit was sent directly to the lab (Quest Diagnostics, Chantilly, VA) and processed on the same day or overnight. The sample was not frozen. The variance in the timing was relatively small since a courier retrieved the samples from our field site and had them delivered to the Quest Diagnostics laboratory facility directly, using the same schedule each day. As stated earlier, several blood biomarkers tend to increase during an inflammation while others tend to decrease [8]. While serum hsCRP and serum fibrinogen tend to increase, serum albumin and transferrin saturation tend to decrease with inflammation [8]. Proxies for serum fibrinogen and transferrin saturation are ESR and serum iron, respectively. Using NHANES III data, the high correlation between serum iron and serum transferrin saturation was verified $(r=0.92, P<0.001)$ in a comparable sample (age range: $30-65 \mathrm{y}$ ). Similarly, previous studies show that ESR is highly correlated with serum fibrinogen [7]. Examining them individually, each marker was used as a continuous untransformed variable in the main analysis. By running principal components analysis, data on the 4 individual measures of inflammation (hsCRP, ESR, albumin and iron) were reduced into a single standardized measure explaining $>40 \%$ of the total variance, which we labelled "z-inflammation composite score" (ICS). Alternative PCA analyses, which included white blood cell counts, serum ferritin and transferrin saturation among others confirmed that this ICS was the most reflective of systemic inflammation and explained the largest proportion of the total variance in a single component. Using the comparable NHANES III data (Age range: 30-65y), a similar PCA analysis substituting serum ESR with serum fibrinogen replicated component loading magnitudes and percentage of variance explained. This ICS component score was also constructed previously [26].

Using refrigerated whole blood $(5 \mathrm{ml})$ stored in EDTA buffer, ESR was tested within $24 \mathrm{~h}$ of blood draw using automated modified Westergren photochemical capillary stopped flow kinetic analysis. Based on the Mayo clinic reports, ESR's reference range is $0-22 \mathrm{~mm} / \mathrm{hr}$ for men and 0-29 mm/hr for women (https://www.mayoclinic.org/testsprocedures/sed-rate/about/pac-20384797). Similarly, hsCRP was analyzed with an immunoturbidimeter (Siemens/Behring Nephelometer II), utilizing $0.5-1 \mathrm{ml}$ of plasma. A range of
$1-3 \mathrm{mg} / \mathrm{dL}$ suggests average and $3-10 \mathrm{mg} / \mathrm{dL}$ high cardiovascular risk while $\mathrm{hsCRP}>10 \mathrm{mg} / \mathrm{dL}$ reflects putative infection or chronic inflammation. Moreover, after collecting $0.5-1 \mathrm{ml}$ sample of plasma prepared with heparin and refrigerated for $<30$ days, albumin was measured using spectrophotometry (expected reference range: $3.6-5.1 \mathrm{~g} / \mathrm{dL}$ ). Finally, $0.5-1 \mathrm{ml}$ of fasting serum was collected for iron determination, which was transported at room temperature (with heparin added), followed by refrigeration or freezing. Serum iron was determined with spectrophotometry, and reference ranges are age and sex-specific, [Men aged $\geq 30 \mathrm{y}: 50-180 \mu \mathrm{g} /$ $\mathrm{dL}$; Women aged 20-49 y (40-190 $\mu \mathrm{g} / \mathrm{dL})$ and women aged $50+\mathrm{y}(45-160 \mu \mathrm{g} / \mathrm{dL})]$.

\section{Cytokines}

All available cytokines were used in our present study. Blood sample aliquots were frozen at $-80{ }^{\circ} \mathrm{C}$ and stored for later use. Interleukin (IL) cytokines were assayed in one batch by Aushon (https://www.aushon.com/) Ciraplex ${ }^{\circledR}$ ULTRA Ultrasensitive Assays in femtogram $/ \mathrm{ml}$ (fg/ml) detection levels. The serum samples were collected from HANDLS participants for studies of DNA repair and agerelated microRNA changes [27, 28]. Cytokines selected for this study included IL-1 $\beta$, IL-6, IL-10, IL-12, and IL-18 (in $\mathrm{pg} / \mathrm{ml}$ ), as they were previously linked with depressive symptoms $[1,2,5]$.

\section{Covariates}

\section{Sociodemographic, lifestyle, and health-related potential confounders}

All models were adjusted for sociodemographic factors, namely age, sex, race (White $v s$. AA), educational attainment categories ( $0 \leq$ High School (HS); $1=$ HS and $2 \geq \mathrm{HS}$ ) and poverty status (below vs. above $125 \%$ the federal poverty line). Poverty status was categorized as such by using the US Census Bureau poverty thresholds for 2004 [29] relying on income, and total family size including children under age 18 years. Moreover, all models with the exception of those with cytokines were further adjusted for measured body mass index $\left(\mathrm{kg} / \mathrm{m}^{2}\right)$, current use of drugs ("opiates, marijuana or cocaine" $=1 v s$. not $=0$ ), and current smoking status (0: "never or former smoker" vs. 1 "current smoker") without examining exposure-covariate associations. Those models were also adjusted for first-visit self-reported history of type 2 diabetes, hypertension, dyslipidemia, cardiovascular disease (stroke, congestive heart failure, non-fatal myocardial infarction or atrial fibrillation), inflammatory disease (multiple sclerosis, systemic lupus, gout, rheumatoid arthritis, psoriasis, thyroid disorder and Crohn's disease) and use of NSAIDs (prescription and 
over-the-counter) over the past two weeks, as was done in previous studies [30, 31]. In models with cytokines, only sociodemographic covariates (age, sex, race, poverty status, education, employment status) and those that were deemed associated with the cytokines in a separate bivariate linear regression model were included.

\section{Dietary potential confounders}

Potential dietary confounders were considered in all models, being previously linked to reduced risk for depression, namely vitamins B- 6 , folate and B-12, total carotenoids $(\alpha-$ carotene, $\beta$-carotene, $\beta$-cryptoxanthin, lutein + zeaxanthin, lycopene), vitamin $C$ and $\alpha$-tocopherol [32-40] (expressed per $1,000 \mathrm{kcal}$ ) and the ratio of $n-3$ polyunsaturated fatty acids (PUFA): $n-6$ PUFA [41]. To emulate a multivariable nutrient density model, energy intake was entered as a covariate [42]. A measure of overall dietary quality, the Healthy Eating Index (HEI-2010) total score, (http://a ppliedresearch.cancer.gov/tools/hei/tools.html and http://ha ndls.nih.gov/06Coll-dataDoc.htm) was also considered. Those covariates were included only with cytokines if they were associated with those exposures (OSM 1).

\section{Statistical analysis}

All analyses were conducted using Stata 15.0 (StataCorp, College Station, TX) [43]. Baseline characteristics, including covariates and exposures, were compared across sex, race and by EDS status (CES-D score $\geq 16$ vs. $<16$, based on mean score across waves), using t-tests and ANOVA for continuous variables and $\chi^{2}$ tests for categorical variables. Second, several linear mixed-effects regression models on continuous CES-D total or on domain-specific score(s) were conducted to test associations with systemic markers of inflammation, including the ICS and cytokines, controlling for potential confounders. The time metric used was TIME elapsed since baseline visit (i.e. visit 1 ) in years. Visit 1 TIME was set at zero while Visit 2 TIME was set at the number of years elapsed, using age difference between visits 1 and 2. Cross-sectional relationships between baseline inflammation and baseline depressive symptoms were estimated using the main fixed effects of each inflammatory marker on the outcome of interest $\left(\gamma_{01 \mathrm{a}}\right.$ for $\left.\pi_{0 \mathrm{i}}\right)$. Longitudinal relationships between baseline inflammation and annual rate of change in depressive symptoms $(\Delta)$ was estimated using the interaction fixed effects between TIME and each of those inflammatory markers $\left(\gamma_{11 \mathrm{a}}\right.$ for $\left.\pi_{1 \mathrm{i}}\right)$. The methodology used is outline in OSM 2 [44-46]. Sex- and race-specific associations were tested by adding interaction terms to the multivariable mixed-effects regression models and stratifying by sex and race, separately. Third, a series of parametric survival models, assuming a Weibull baseline hazard function form, examined the association between baseline ICS and incident EDS, stratifying by sex and race [47]. Hazard ratios with $95 \%$ CIs were estimated for each SD increase in ICS in relation to instantaneous adjusted hazard of incident EDS, defined as a transition into a total CES-D score from $<16$ to $\geq 16$ or higher over time. Cox proportional hazards models were also carried out as a sensitivity analysis [47].

Non-random selection of participants from the initial probability HANDLS sample $(n=3,720)$ may cause bias due to systematic differentials in basic characteristics such as age, sex, race and socio-economic status between the final analytic sample and the excluded sample. A 2-stage Heckman process accounted for selection bias in all final models. At the first stage, a probit model with a binary outcome variable coded as selected $=1 v s$. unselected $=0$ was conducted from which an inverse mills ratio (derived from the predicted probability of being selected, conditional on the covariates baseline age, sex, race, poverty status and education) was estimated. At a second stage, this inverse mills ratio was entered into each mixed-effects regression model as a covariate, as was done in prior studies [48]. Two inverse mills ratio were computed, one for the ICS subsample and one for the cytokine sub-sample.

A type I error of 0.05 was used, with $0.05<p$-values $<$ 0.10 judged as borderline significant for main effects, while $p$-value $<0.10$ was considered significant for interaction terms [49] before family-wise Bonferroni correction for multiple testing [50], assuming CES- $\mathrm{D}_{\text {total }}$ and sub-domain scores are distinctive outcomes, while the 10 exposures that are conceptually related. This approach was adopted in several previous studies [46, 51]. Accounting for 10 exposures, type I error was reduced to $0.05 / 10=0.005$ for main effects and $0.10 / 10=0.010$ for interaction terms for the mixed-effects regression models, with no adjustment done when ICS was tested in the parametric survival models.

\section{Results}

Table 1 displays study sample characteristics across sex, race and EDS status. Notably, mean CES-D was higher among women, as were means of hsCRP and ESR. The reverse was found for serum albumin and iron leading to a higher ICS among women. Similarly, AA and $\mathrm{EDS}^{+}$participants had higher mean ICS compared with Whites and EDS $^{-}$individuals, respectively. Within the smaller subsample having cytokine data, a higher mean IL-6 was detected among $\mathrm{EDS}^{+}$compared to $\mathrm{EDS}^{-}$individuals. Sex differences in EDS status was also noted, whereby $46.7 \%$ of $\mathrm{EDS}^{-}$were men (vs. $36.0 \%$ of $\mathrm{EDS}^{+}$), suggesting a higher prevalence among women. Educational attainment beyond high school was more prevalent among Whites and EDS ${ }^{-}$ 


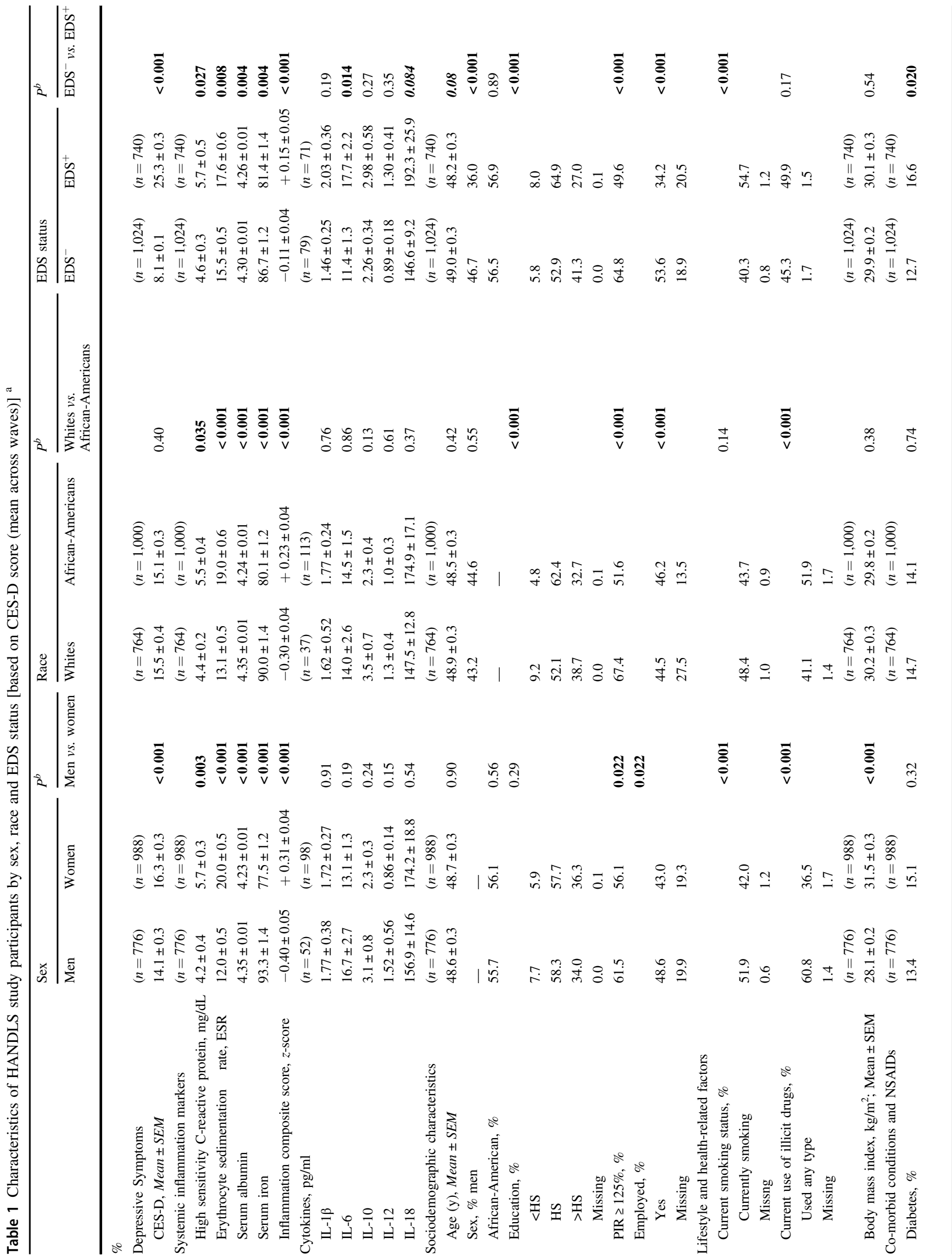




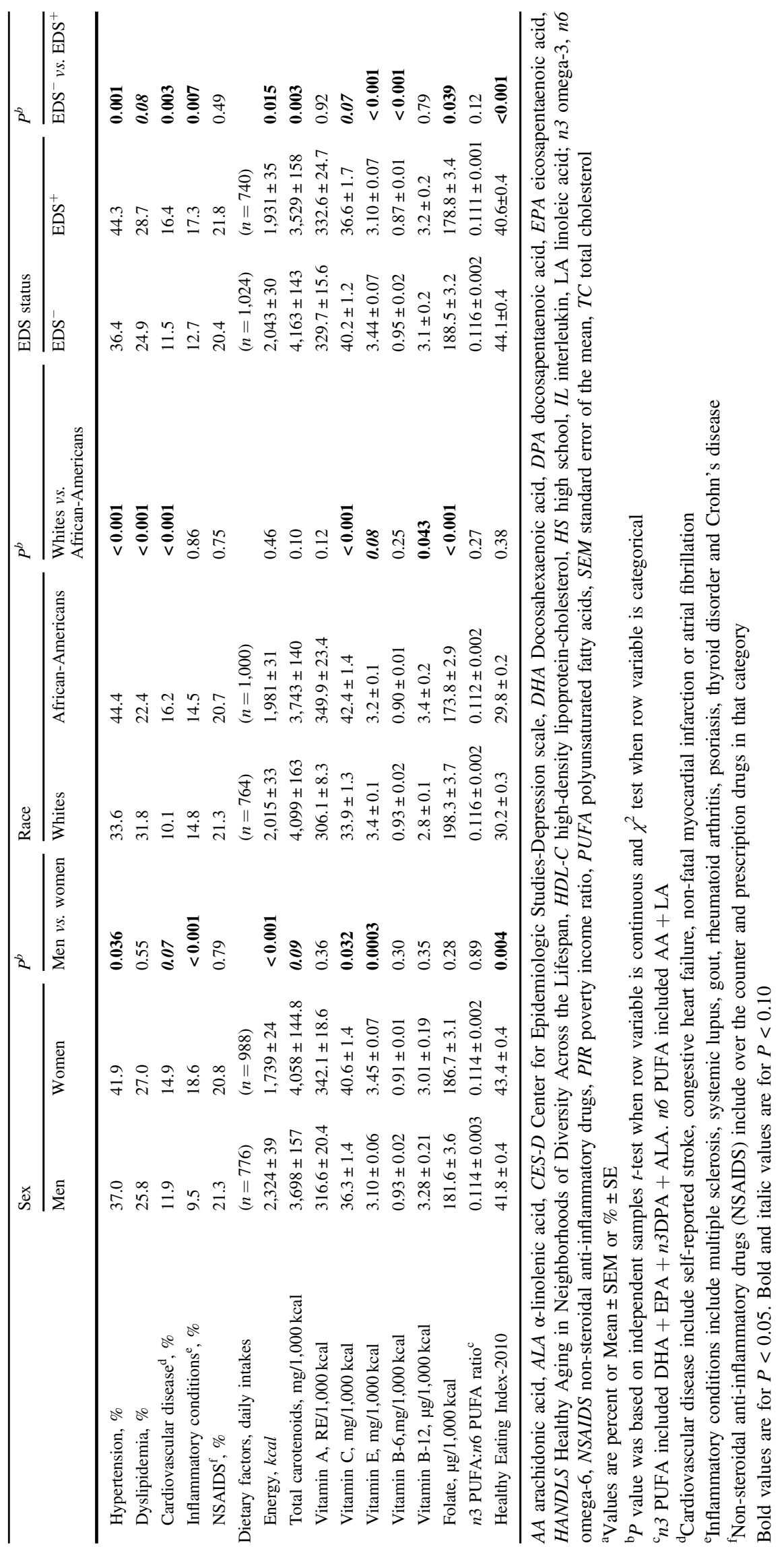


individuals, compared with $\mathrm{AA}$ and $\mathrm{EDS}^{+}$participants. A greater prevalence of employment and "above poverty" status was also observed among men, Whites and EDS ${ }^{-}$ individuals. Men were more likely current smokers and drug users, as were $\mathrm{EDS}^{+}$individuals compared to women and $\mathrm{EDS}^{-}$counterparts, respectively. Illicit drug use was also more prevalent among AA compared to Whites. Mean BMI and prevalence of hypertension and inflammatory conditions were higher among women, while among AA, hypertension and cardiovascular disease were more prevalent compared with Whites, with a reverse pattern by race observed for dyslipidemia. Similarly, $\mathrm{EDS}^{+}$individuals had a higher prevalence of type 2 diabetes, hypertension, cardiovascular disease and inflammatory conditions. While HEI-2010 indicated better overall dietary quality in women and $\mathrm{EDS}^{-}$individuals, other micronutrients exhibited different patterns across sex, race and EDS status. Most notably, total carotenoids, vitamins E, B-6 and folate (per $1,000 \mathrm{kcal})$ were higher among $\mathrm{EDS}^{-}$individuals compared to the $\mathrm{EDS}^{+}$groups. Folate intake per $1,000 \mathrm{kcal}$ was also higher among Whites but the reverse pattern by race was true for vitamin B-12 and C intakes per $1000 \mathrm{kcal}$.

Using mixed-effects linear regression models (OSM 2), we examined the associations of systemic inflammatory markers and cytokines with baseline and longitudinal annual rate of change in depressive symptoms $(\Delta)$, while stratifying our analyses by sex and race, separately. Table 2 focuses on the total CES-D score and Table 3 shows results for each of the 4 CES-D component scores. After correction for multiple testing, and among Whites only, hsCRP, ICS and IL-1 $\beta$ were all associated with a faster $\Delta$ CES-D total, with significant racial differences. The result for ICS among Whites is illustrated as predictive margins of CES- $\mathrm{D}_{\text {total }}$ across $1 \mathrm{SD}$ changes in ICS in Fig. 1. This Figure shows clearly that the rate of change in CES- $\mathrm{D}_{\text {total }}$ over time is increased with each higher level of ICS among Whites. In contrast, IL-12 was directly associated with a faster $\triangle \mathrm{CES}$ $\mathrm{D}_{\text {total }}$ though only among men and AA, with significant sex and race differentials (Table 2). The race-specific associations of hsCRP, ICS, IL- $1 \beta$ and the sex-specific association of IL-12 with $\triangle$ CES-D trajectory were consistently detected for the "depressed affect" domain (component 2) (Table 3). For component 1 of CES-D, and among men only, lower serum albumin and higher ICS were both associated with a higher score suggesting greater "somatic complaints" at baseline. The third component of the CES-D ("positive affect") was longitudinally associated with IL-10 among AA and IL-12 among men, suggesting that those cytokines were specifically linked to reducing positive affect over time in those population groups. Finally, the fourth component of the CES-D ("interpersonal problems") was crosssectionally associated with IL-6 among AA and IL-10 among Whites.
Table 4 shows results of the association between ICS and incident EDS, across sex and race groups. Out of 1,259 subjects who were EDS-free at baseline in HANDLS, 305 incidence EDS cases were observed over a median follow-up period of $4.71 \mathrm{y}$. In the final analytic sample, EDS-free subjects were $N=796$ of whom 185 became incident EDS cases. Parametric survival models with Weibull hazard function distribution revealed that a unit (i.e., $1 \mathrm{SD}$ ) increase in the ICS was associated with a $28 \%$ higher risk of incident $\mathrm{EDS}(\mathrm{HR}=1.28,95 \% \mathrm{CI}: 1.04-1.56, P=$ 0.017) among AA only. A similar estimate was obtained from Cox PH models, while no other sex/race strata showed a significant link between ICS and incident EDS.

\section{Discussion}

This study tested the association between systemic inflammation and depressive symptoms in a prospective bi-racial cohort of urban adults. Findings suggest that serum concentrations of high-sensitivity C-reactive protein (hsCRP), z-inflammation composite score [ICS, combining elevated hsCRP and ESR with low serum albumin and iron], and serum interleukin IL- $1 \beta$ were positively associated with $\Delta$ CES-Dtotal ( $\Delta$ : annual rate of increase) among Whites only. IL-12 was directly related to $\Delta$ CES-Dtotal among men and AA. The race-specific associations of hsCRP, ICS, IL-1 $\beta$ and the sex-specific association of IL-12 with $\Delta$ CESDtotal were replicated for the "depressed affect" domain. Among men, lower serum albumin and higher ICS were linked with higher baseline "somatic complaints". IL-10 among AA and IL-12 among men were inversely related to $\Delta$ "positive affect", while "interpersonal problems" were cross-sectionally associated with IL-6 among AA and IL-10 among Whites. Finally, baseline ICS was positively associated with incident "elevated depressive symptoms" (EDS: CES-Dtotal $\geq 16)$ among AA $(\mathrm{HR}=1.28$, 95\% CI: $1.04-$ 1.56, $P=0.017)$.

Large epidemiologic studies published between 2004 and 2018, have found inconsistent results pertaining to associations between CRP and depression. In a longitudinal study among 5,810 participants from the Great Smoky Mountains Study, Copland et al. provided evidence for depression as a predictor of later CRP levels, but not CRP as a predictor of later depression [52]. In a cross-sectional study of 512 Morehouse and Emory Team up to Eliminate Health Disparities participants, Morris et al. found an association between CRP and the Beck Depression Inventory in White women only and this association was mediated partly by waist circumference [13]. Analyses of data from the Pittsburgh Healthy Heart Project $(N=263)$ found a weak bidirectional relationship between CRP and Beck Depression Inventory-II [11]. Furthermore, based on the 


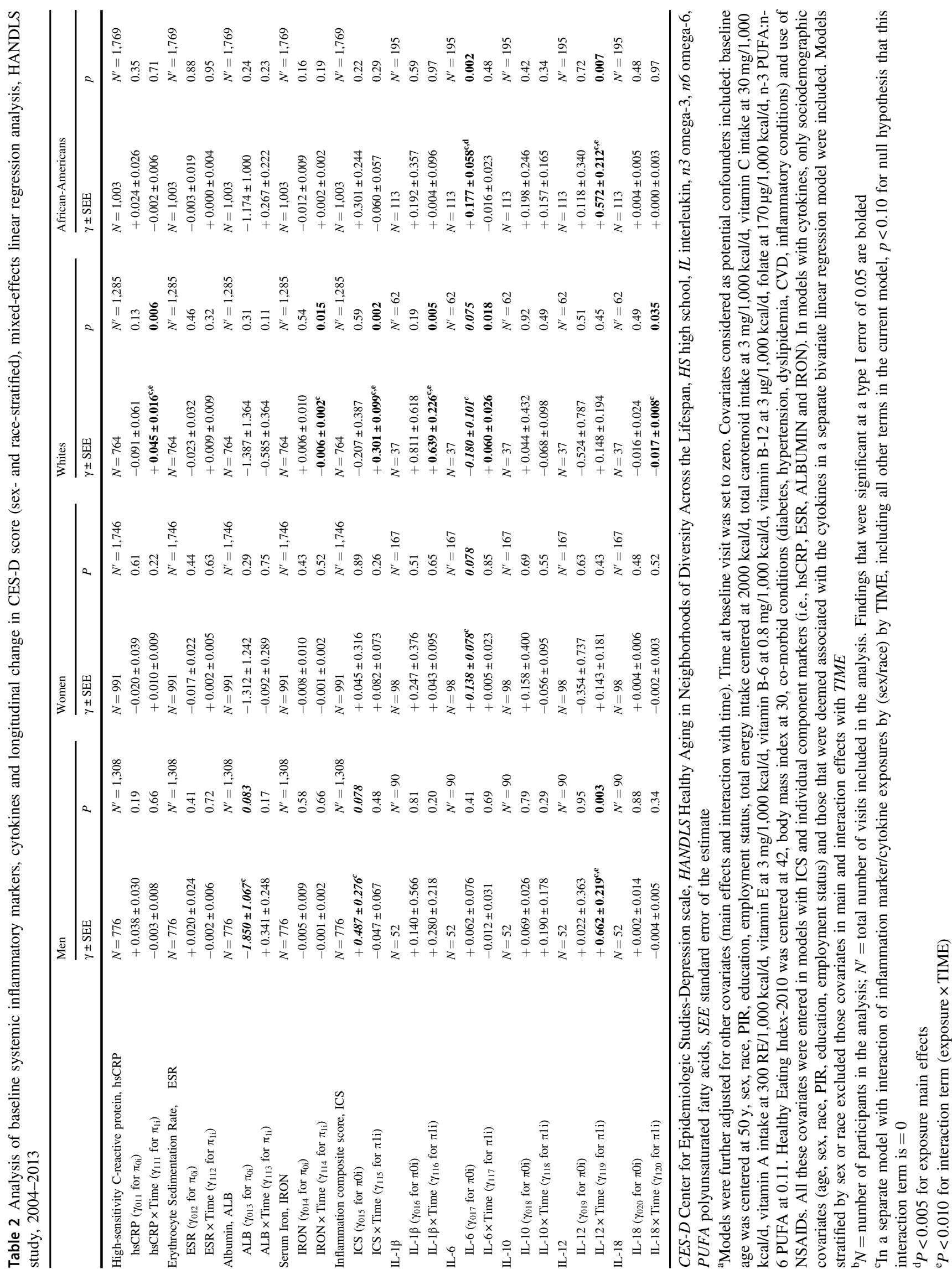




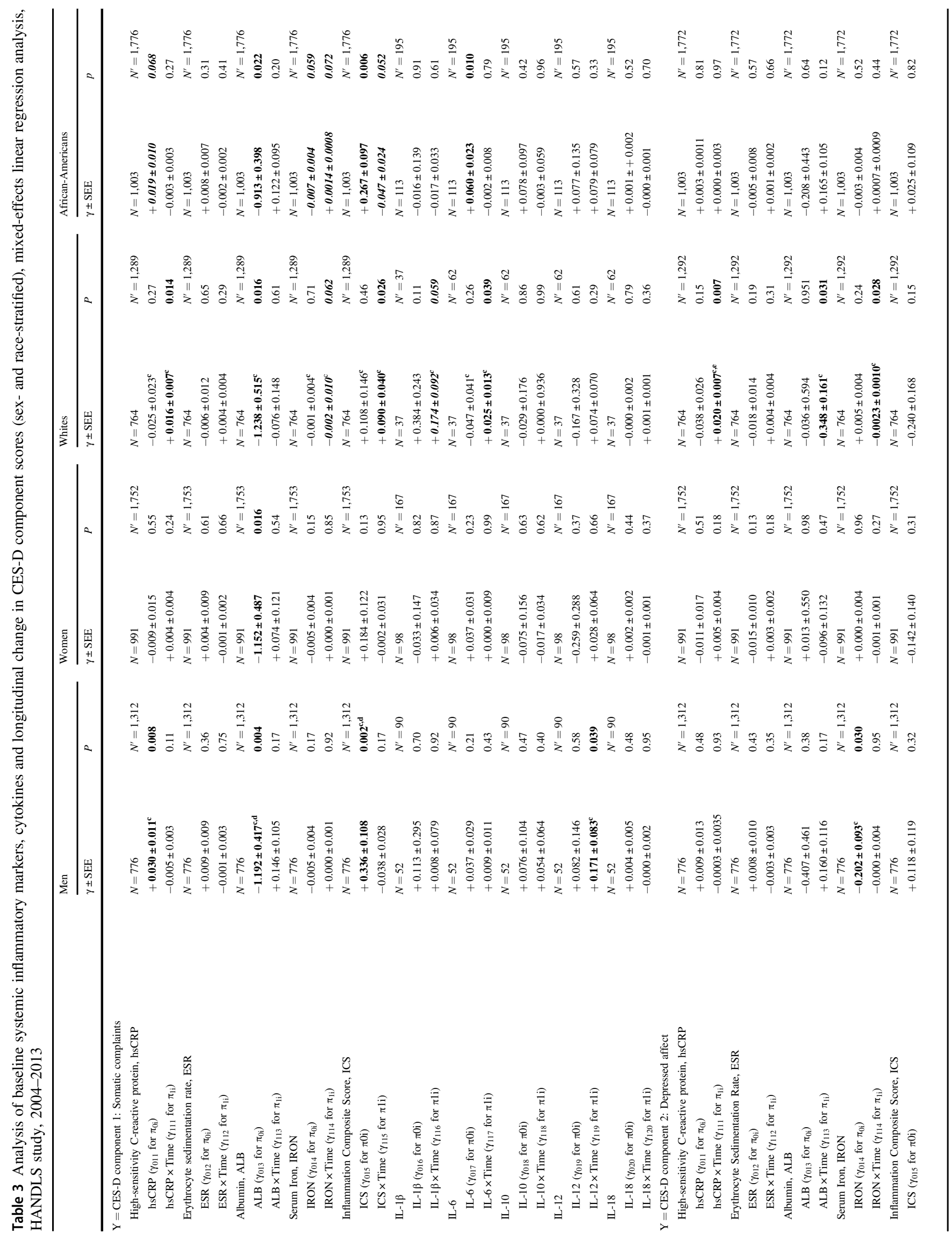




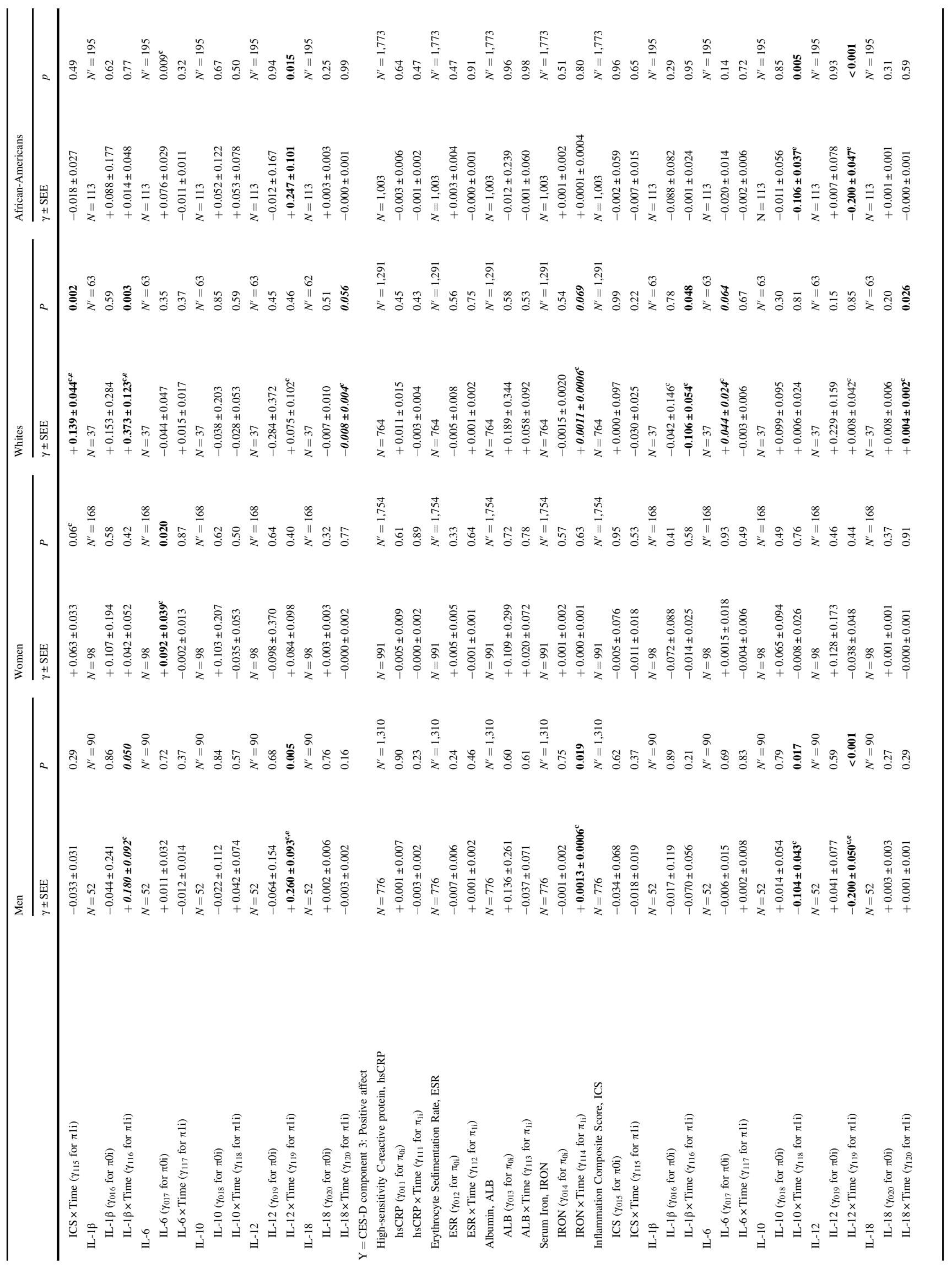




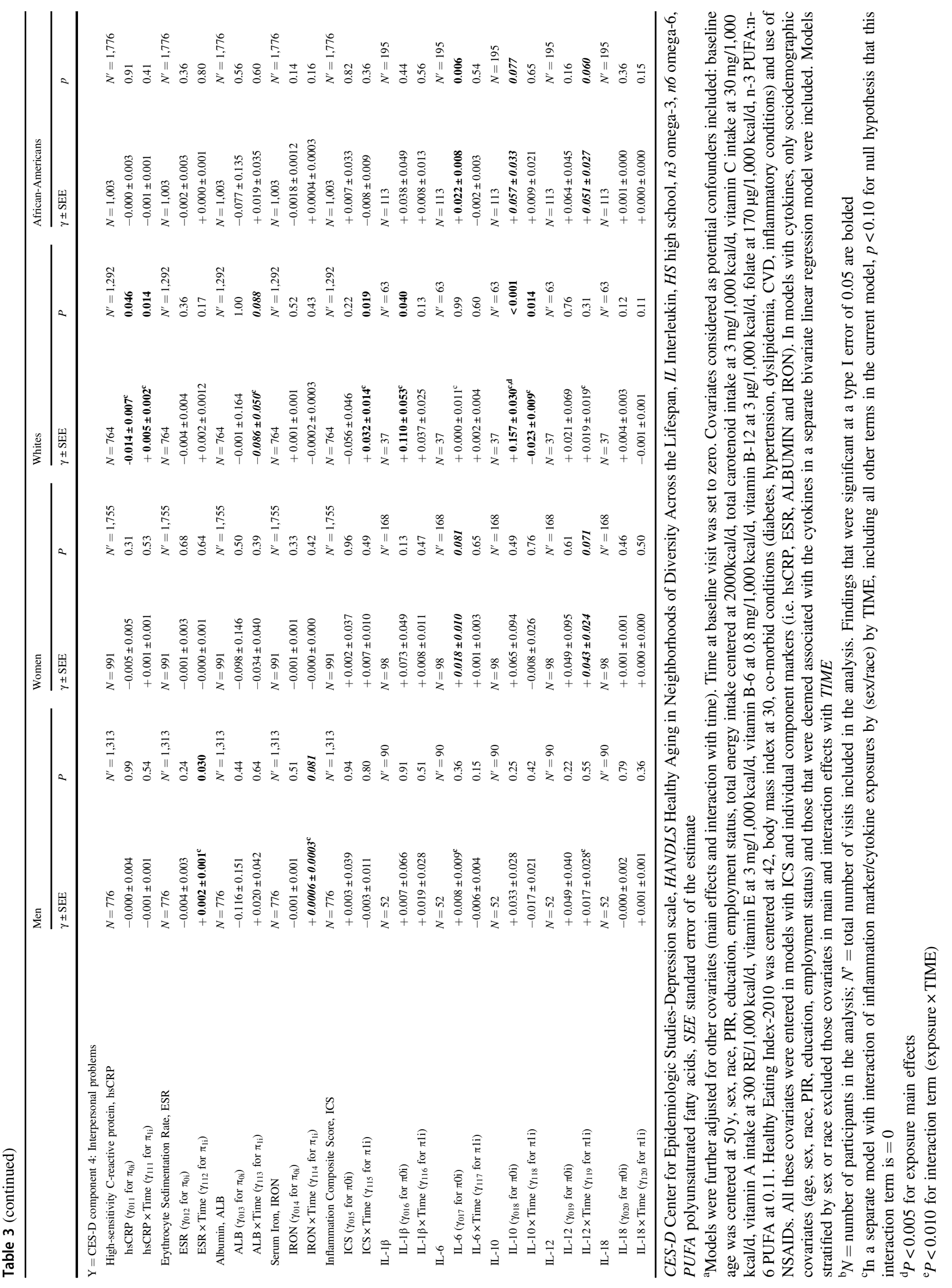


ATTICA cross-sectional study involving 853 individuals, a positive correlation was observed between the Zung SelfRating Depression Scale and CRP [3]. Using data on 1,167 male subjects from the Men Androgen Inflammation Lifestyle Environment and Stress (MAILES) Study (Australia, Asia, Europe), Tully et al. found hsCRP was associated with depression (BDI-I) and increased depressive symptoms (CES-D) in men without abdominal obesity nor metabolic risk [16]. Duivis et al. examined the longitudinal relationship between hsCRP and depressive symptoms among 667 participants from the Heart and Soul Study and found no bidirectional relationship between inflammation and depression [12]. In the longitudinal Geelong Osteoporosis Study (Australia), Pasco et al. found a direct association between hsCRP and diagnosis of MDD [53]. Other inflammatory

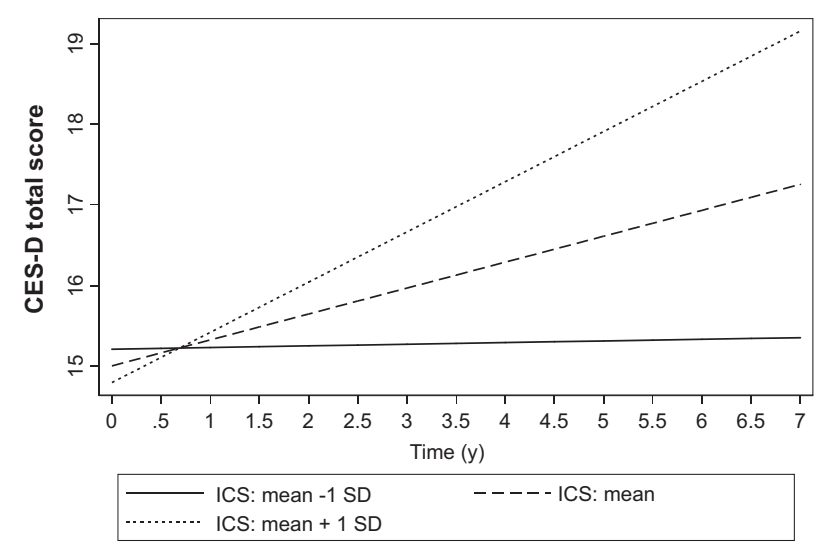

Fig. 1 Predictive margins of CES-D total score by ICS among Whites from multiple linear mixed-effects regression model, HANDLS 2004-2013 markers besides CRP and hsCRP were also examined in relation to depression. 53 In a cross-sectional study, alHakeim et al. found significant increases in pro-inflamatory cytokines IL-6, IL-18, TNF $\alpha$, and sIL-2R in patients diagnosed with MDD compared with the control group [15]. Finally, using data on 5978 subjects the Whitehall II study of British civil servants, Gimeno et al. found that baseline CRP and IL-6 were associated with cognitive symptoms of depression (General Health Questionnaire) at follow-up [30]. Thus, given the paucity of longitudinal studies that examined trajectories of depressive symptoms in relation to inflammatory markers and cytokines, our study adds to the body of evidence showing that in fact baseline inflammation can trigger worsening of depressive symptoms over time.

Cytokines can influence depression by dysregulating neurotransmitter synaptic availability of monoamines such as serotonin, noradrenaline and dopamine, as well as the metabolism of various amino acids such as tyrosine, tryptophan, phenylalanine and glutamate [54]. For instance, IL$1 \beta$ and TNF- $\alpha$ can induce p38 mitogen-activated protein kinase (MAPK), which in turn can increase expression and function of serotonin reuptake pumps, leading to decreased serotonin synaptic availability leading ultimately to depressive-like behavior in laboratory animals [55]. By generating reactive oxygen or nitrogen species, proinflammatory cytokines can also decrease the availability of tetrahydrobiopterin (BH4), a key enzyme co-factor in monoamine synthesis [56]. In fact, $\mathrm{BH} 4 \mathrm{CSF}$ concentrations were inversely related to IL-6 CSF concentrations among patients treated with the IFN- $\alpha$ inflammatory cytokine [57]. Moreover, activation of indoleamine 2,3 dioxygenase (IDO) enzyme may be involved in cytokine-induced
Table 4 Parametric survival model, with Weibull hazard distribution, for incident EDS $v s$. baseline ICS, HANDLS 20042013

\begin{tabular}{lllllll}
\hline & $\mathrm{N}^{\mathrm{b}}$ & $\mathrm{n}^{\mathrm{b}}$ & $\mathrm{T}^{\mathrm{b}}$ & Hazard Ratio & $(95 \% \mathrm{CI})$ & $P$ \\
\hline ICS & & & & & & \\
All & 796 & 185 & 3,621 & $\mathbf{1 . 1 5}$ & $\mathbf{( 0 . 9 9 - 1 . 3 4 )}$ & $\mathbf{0 . 0 6 8}$ \\
Men & 348 & 66 & 1,605 & 1.11 & $(0.86-1.43)$ & 0.44 \\
Women & 448 & 119 & 2,017 & $\mathbf{1 . 2 0}$ & $\mathbf{( 0 . 9 8 - 1 . 4 7 )}$ & $\mathbf{0 . 0 7 3}$ \\
Whites & 330 & 83 & 1,450 & 1.10 & $(0.86-1.41)$ & 0.45 \\
African-Americans & 466 & 102 & 2,171 & $\mathbf{1 . 2 8}$ & $\mathbf{( 1 . 0 4 - 1 . 5 6 )}$ & $\mathbf{0 . 0 1 7}$ \\
\hline
\end{tabular}

CES-D Center for Epidemiologic Studies-Depression scale, HANDLS healthy aging in neighborhoods of diversity across the lifespan, $H S$ high school, ICS inflammation composite Score, $n 3$ omega-3, $n 6$ omega-6, PUFA polyunsaturated fatty acids, SEE standard error of the estimate

${ }^{a}$ Models were further adjusted for other covariates. Covariates considered as potential confounders included: baseline age was centered at $50 \mathrm{y}$, sex, race, PIR, education, employment status, total energy intake centered at $2000 \mathrm{kcal} / \mathrm{d}$, total carotenoid intake at $3 \mathrm{mg} / 1,000 \mathrm{kcal} / \mathrm{d}$, vitamin $\mathrm{C}$ intake at $30 \mathrm{mg} / 1,000 \mathrm{kcal} / \mathrm{d}$, vitamin A intake at $300 \mathrm{RE} / 1,000 \mathrm{kcal} / \mathrm{d}$, vitamin $\mathrm{E}$ at $3 \mathrm{mg} / 1,000 \mathrm{kcal} / \mathrm{d}$, vitamin B-6 at $0.8 \mathrm{mg} / 1,000 \mathrm{kcal} / \mathrm{d}$, vitamin B-12 at $3 \mu \mathrm{g} / 1,000 \mathrm{kcal} / \mathrm{d}$, folate at $170 \mu \mathrm{g} / 1,000 \mathrm{kcal} / \mathrm{d}, \mathrm{n}-3$ PUFA:n-6 PUFA at 0.11 . Healthy Eating Index2010 was centered at 42, body mass index at 30, co-morbid conditions (diabetes, hypertension, dyslipidemia, CVD, inflammatory conditions) and use of NSAIDs. Models stratified by sex or race excluded those covariates

${ }^{\mathrm{b}} N=$ number of subjects, $n=$ number of failures, $\mathrm{T}=$ time at risk (Person-years)

Bold values are for $P<0.05$. Bold and italic values are for $P<0.10$ 
neurotransmitter alterations, partly through diverting tryptophan metabolism (a precursor of serotonin) into kynurenine, which converts to quinolinic acid, a neurotoxin produced by the activated microglia, monocytes and macrophages infiltrating the brain $[58,59]$. High concentrations of quinolinic acid were found in microglia of the anterior cingulate gyrus (ACC) among suicide victims who suffered from depression [60]. Quinolinic acid directly activates the $\mathrm{N}$-methyl-d-aspartate (NMDA) receptors while also stimulating glutamate release by blocking glutamate synaptic reuptake in astrocytes [61, 62]. Glutamate's binding to extrasynaptic NMDA receptors can increase excitotoxicity while reducing brain-derived neurotrophic factor (BDNF) production [63]. BDNF is key to an antidepressant response partly through fostering of neurogenesis. In stress-induced animal models of depression, BDNF was shown to be markedly reduced by IL- $1 \beta$ and TNF- $\alpha$ and their downstream signaling pathways including NF-кB [64, 65]. Increased basal ganglia and dorsal ACC glutamate levels have been described in patients receiving IFN- $\alpha$, while higher glutamate concentrations were associated with elevated depressive symptoms [66]. Recently, hsCRP $>3 \mathrm{mg} / \mathrm{L}$ was associated with increased basal ganglia glutamate $(v s$. a hsCRP $<$

$1 \mathrm{mg} / \mathrm{L}$ ) that correlated with anhedonia and poor psychomotor speed among depressed patients [67].

The study has several notable strengths. First, its longitudinal design improves the ability to ascertain temporal relationships between the exposure of systemic inflammation and the outcome of depressive symptoms. Second, the large final analytic sample size allowed for further stratification by key sociodemographic factors, namely sex and race without significant decline in statistical power. Third, the intercept and slopes in the mixedeffects regression models were adjusted for numerous potentially confounding covariates. Nevertheless, several limitations should also be noted, such as potential nonparticipation selection bias, the effect of which was minimized by the use of a 2-stage Heckman selection model. Furthermore, while inflammatory markers were direct blood level measures, our outcome of interest was self-report which could be a source of measurement error. Given the observational nature of the study, residual confounding cannot be ruled out. Finally, although no bidirectional analysis was done, this can potentially be carried out in a future study.

\section{Conclusion}

In sum, several markers of systemic inflammation and cytokines were shown to be linked to depressive symptoms' trajectory over time, differentially across sex and race groups, with each exposure being predictive of baseline and rates of change of specific depressive symptom domains. While a composite score of inflammation (ICS) was found to be linked with a faster trajectory of CES-D scores and domains for the most part among Whites, ICS was also directly related to incident EDS among AA. Given those inconsistent race-specific findings between annual rates of change $v s$. incident binary outcomes, additional longitudinal studies in similar large and diverse samples are needed.

Acknowledgements This work was supported by the Intramural Research Program of the National Institutes of Health, National Institute on Aging, NIA/NIH/IRP. J. Weiss received support from the Population Research Training Grant (NIH T32 HD007242) awarded to the Population Studies Center at the University of Pennsylvania by the National Institutes of Health's (NIH)'s Eunice Kennedy Shriver National Insitute of Child Health and Human Development. It was also supported by the NIH/NIA under grant F31AG058370 awarded to H.K. Obhi.

Funding This research was supported by the Intramural Research Program of the NIH, National Institute on Aging.

Author contributions MAB had full access to the data used in this manuscript and completed all the statistical analyses.

\section{Compliance with ethical standards}

Conflict of interest The authors declare that they have no conflict of interest.

Disclaimer The views expressed in this article are those of the author (s) and do not reflect the official policy of the Department of the Army/ Navy/Air Force, Department of Defense, or the U.S. Government.

Publisher's note: Springer Nature remains neutral with regard to jurisdictional claims in published maps and institutional affiliations.

Open Access This article is licensed under a Creative Commons Attribution 4.0 International License, which permits use, sharing, adaptation, distribution and reproduction in any medium or format, as long as you give appropriate credit to the original author(s) and the source, provide a link to the Creative Commons license, and indicate if changes were made. The images or other third party material in this article are included in the article's Creative Commons license, unless indicated otherwise in a credit line to the material. If material is not included in the article's Creative Commons license and your intended use is not permitted by statutory regulation or exceeds the permitted use, you will need to obtain permission directly from the copyright holder. To view a copy of this license, visit http://creativecommons. org/licenses/by/4.0/.

\section{References}

1. Miller AH, Raison CL. The role of inflammation in depression: from evolutionary imperative to modern treatment target. Nat Rev Immunol. 2016;16:22-34.

2. Zunszain PA, Hepgul N, Pariante CM. Inflammation and depression. Curr Top Behav Neurosci. 2013;14:135-51.

3. Panagiotakos DB, Pitsavos C, Chrysohoou C, Tsetsekou E, Papageorgiou C, Christodoulou $\mathrm{G}$, et al. Inflammation, 
coagulation, and depressive symptomatology in cardiovascular disease-free people; the ATTICA study. Eur Heart J. 2004;25: 492-9.

4. Ariyo AA, Haan M, Tangen CM, Rutledge JC, Cushman M, Dobs A, et al. Depressive symptoms and risks of coronary heart disease and mortality in elderly Americans. Cardiovascular Health Study Collaborative Research Group. Circulation. 2000;102:1773-9.

5. Miller AH, Maletic V, Raison CL. Inflammation and its discontents: the role of cytokines in the pathophysiology of major depression. Biol Psychiatry. 2009;65:732-41.

6. Black S, Kushner I, Samols D. C-reactive protein. J Biol Chem. 2004;279:48487-90.

7. Talstad I, Haugen HF. The relationship between the erythrocyte sedimentation rate (ESR) and plasma proteins in clinical materials and models. Scand J Clin Lab Invest. 1979;39:519-24.

8. Gabay C, Kushner I. Acute-phase proteins and other systemic responses to inflammation. N Engl J Med. 1999;340:448-54.

9. Kop WJ, Gottdiener JS, Tangen CM, Fried LP, McBurnie MA, Walston $J$, et al. Inflammation and coagulation factors in persons $>65$ years of age with symptoms of depression but without evidence of myocardial ischemia. Am J Cardiol. 2002;89: 419-24.

10. Kiecolt-Glaser JK, Belury MA, Porter K, Beversdorf DQ, Lemeshow S, Glaser R. Depressive symptoms, omega-6:omega-3 fatty acids, and inflammation in older adults. Psychosom Med. 2007;69:217-24.

11. Stewart JC, Rand KL, Muldoon MF, Kamarck TW. A prospective evaluation of the directionality of the depression-inflammation relationship. Brain Behav Immun. 2009;23:936-44.

12. Duivis HE, de Jonge P, Penninx BW, Na BY, Cohen BE, Whooley MA. Depressive symptoms, health behaviors, and subsequent inflammation in patients with coronary heart disease: prospective findings from the heart and soul study. Am J Psychiatry. 2011;168:913-20.

13. Morris AA, Zhao L, Ahmed Y, Stoyanova N, De Staercke C, Hooper WC, et al. Association between depression and inflammation-differences by race and sex: the META-Health study. Psychosom Med. 2011;73:462-8.

14. Perez-Cornago A, de la Iglesia R, Lopez-Legarrea P, Abete I, Navas-Carretero S, Lacunza CI, et al. A decline in inflammation is associated with less depressive symptoms after a dietary intervention in metabolic syndrome patients: a longitudinal study. Nutr J. 2014;13:36.

15. Al-Hakeim HK, Al-Rammahi DA, Al-Dujaili AH. IL-6, IL-18, sIL-2R, and TNFalpha proinflammatory markers in depression and schizophrenia patients who are free of overt inflammation. J Affect Disord. 2015;182:106-14.

16. Tully PJ, Baumeister H, Bengel J, Jenkins A, Januszewski A, Martin S, et al. The longitudinal association between inflammation and incident depressive symptoms in men: the effects of hs-CRP are independent of abdominal obesity and metabolic disturbances. Physiol Behav. 2015;139:328-35.

17. Mezuk B, Choi M, DeSantis AS, Rapp SR, Diez Roux AV, Seeman T. Loneliness, depression, and inflammation: evidence from the multi-ethnic study of atherosclerosis. PLoS ONE. 2016;11:e0158056

18. Su Q, Gu Y, Yu B, Yu F, He H, Zhang Q, et al. Association between Serum Ferritin Concentrations And Depressive Symptoms Among Chinese Adults: A Population Study From The Tianjin Chronic Low-grade Systemic Inflammation And Health (TCLSIHealth) Cohort Study. PLoS ONE. 2016;11:e0162682.

19. Gallagher D, Kiss A, Lanctot K, Herrmann N. Depression with inflammation: longitudinal analysis of a proposed depressive subtype in community dwelling older adults. Int J Geriatr Psychiatry. 2017;32:e18-e24.
20. Herder C, Schmitt A, Budden F, Reimer A, Kulzer B, Roden M, et al. Longitudinal associations between biomarkers of inflammation and changes in depressive symptoms in patients with type 1 and type 2 diabetes. Psychoneuroendocrinology. 2018;91:216-25.

21. Evans MK, Lepkowski JM, Powe NR, LaVeist T, Kuczmarski $\mathrm{MF}$, Zonderman AB. Healthy aging in neighborhoods of diversity across the life span (HANDLS): overcoming barriers to implementing a longitudinal, epidemiologic, urban study of health, race, and socioeconomic status. Ethnicity \& disease. 2010;20:267-75.

22. Radloff L. The CES-D scale: a self-report depression scale for research in the general population. Applied Psychological Measurement. 1977; 1:385-401.

23. Beekman AT, Deeg DJ, Van Limbeek J, Braam AW, De Vries MZ, Van Tilburg W. Criterion validity of the Center for Epidemiologic Studies Depression scale (CES-D): results from a community-based sample of older subjects in The Netherlands. Psychol Med. 1997;27:231-5.

24. Ramos MI, Allen LH, Haan MN, Green R, Miller JW. Plasma folate concentrations are associated with depressive symptoms in elderly Latina women despite folic acid fortification. Am J Clin Nutr. 2004;80:1024-8.

25. Nguyen HT, Kitner-Triolo M, Evans MK, Zonderman AB. Factorial invariance of the CES-D in low socioeconomic status African Americans compared with a nationally representative sample. Psychiatry Res. 2004;126:177-87.

26. Beydoun MA, Dore GA, Canas JA, Liang H, Beydoun HA, Evans MK, et al. Systemic inflammation is associated with longitudinal changes in cognitive performance among urban adults. Front Aging Neurosci. 2018;10:313.

27. Noren Hooten N, Ejiogu N, Zonderman AB, Evans MK. Association of oxidative DNA damage and C-reactive protein in women at risk for cardiovascular disease. Arterioscler Thromb Vasc Biol. 2012;32:2776-84.

28. Noren Hooten N, Fitzpatrick M, Wood WH 3rd, De S, Ejiogu N, Zhang Y, et al. Age-related changes in microRNA levels in serum. Aging. 2013;5:725-40.

29. Bureau UC. US Census Bureau, Social, Economic, and Housing Statistics Division. Poverty Thresholds, 2004. 2014. 2004. Accessed January 2016. https://www.census.gov/data/tables/timeseries/demo/income-poverty/historical-poverty-thresholds.html.

30. Gimeno D, Kivimaki M, Brunner EJ, Elovainio M, De Vogli R, Steptoe A, et al. Associations of C-reactive protein and interleukin6 with cognitive symptoms of depression: 12-year follow-up of the Whitehall II study. Psychol Med. 2009;39:413-23.

31. Bettcher BM, Wilheim R, Rigby T, Green R, Miller JW, Racine $\mathrm{CA}$, et al. C-reactive protein is related to memory and medial temporal brain volume in older adults. Brain Behav Immun. 2012;26:103-8.

32. Morris DW, Trivedi MH, Rush AJ. Folate and unipolar depression. J Altern Complement Med. 2008;14:277-85.

33. Scott TM, Tucker KL, Bhadelia A, Benjamin B, Patz S, Bhadelia $\mathrm{R}$, et al. Homocysteine and $\mathrm{B}$ vitamins relate to brain volume and white-matter changes in geriatric patients with psychiatric disorders. Am J Geriatric Psychiatry. 2004;12:631-8.

34. D'Anci KE, Rosenberg IH. Folate and brain function in the elderly. Curr Opin Clin Nutr Metab Care. 2004;7:659-64.

35. Oishi J, Doi H, Kawakami N. Nutrition and depressive symptoms in community-dwelling elderly persons in Japan. Acta Med Okayama. 2009;63:9-17.

36. Owen AJ, Batterham MJ, Probst YC, Grenyer BF, Tapsell LC. Low plasma vitamin $\mathrm{E}$ levels in major depression: diet or disease? Eur J Clin Nutr. 2005;59:304-6.

37. Maes M, De Vos N, Pioli R, Demedts P, Wauters A, Neels H, et al. Lower serum vitamin $\mathrm{E}$ concentrations in major depression. Another marker of lowered antioxidant defenses in that illness. $\mathbf{J}$ Affect Disord. 2000;58:241-6. 
38. Murakami K, Mizoue T, Sasaki S, Ohta M, Sato M, Matsushita Y, et al. Dietary intake of folate, other B vitamins, and omega-3 polyunsaturated fatty acids in relation to depressive symptoms in Japanese adults. Nutrition. 2008;24:140-7.

39. Kamphuis MH, Geerlings MI, Grobbee DE, Kromhout D. Dietary intake of B(6-9-12) vitamins, serum homocysteine levels and their association with depressive symptoms: the Zutphen Elderly Study. Eur J Clin Nutr. 2008;62:939-45.

40. Cherubini A, Martin A, Andres-Lacueva C, Di Iorio A, Lamponi $\mathrm{M}$, Mecocci P, et al. Vitamin E levels, cognitive impairment and dementia in older persons: the InCHIANTI study. Neurobiol Aging. 2005;26:987-94.

41. Beydoun MA, Tanaka T, Beydoun HA, Ding EL, Ferrucci L, Zonderman $\mathrm{AB}$. Vitamin $\mathrm{D}$ receptor and megalin gene polymorphisms are associated with central adiposity status and changes among US adults. J Nutr Sci. 2013;2:e33.

42. Willet WC. Nutritional epidemiology 2nd ed. New York: Oxford University Press; 1998.

43. STATA.. Statistics/Data Analysis: Release 15.0. Texas: Stata Corporation; 2017.

44. Blackwell E, de Leon CF, Miller GE. Applying mixed regression models to the analysis of repeated-measures data in psychosomatic medicine. Psychosom Med. 2006;68:870-8.

45. Beydoun MA, Gamaldo AA, Beydoun HA, Tanaka T, Tucker KL, Talegawkar SA, et al. Caffeine and alcohol intakes and overall nutrient adequacy are associated with longitudinal cognitive performance among U.S. adults. J Nutr. 2014;144:890-901.

46. Beydoun MA, Beydoun HA, Dore GA, Fanelli-Kuczmarski MT, Evans MK, Zonderman AB. Total serum cholesterol, atherogenic indices and their longitudinal association with depressive symptoms among US adults. Transl Psychiatry. 2015;5:e518.

47. Royston P, Lambert PC. Flexible parametric survival analysis using Stata: beyond the Cox model. TX: Stata Corp. LP: College Station; 2011

48. Beydoun MA, Beydoun HA, Kitner-Triolo MH, Kaufman JS, Evans MK, Zonderman AB. Thyroid hormones are associated with cognitive function: moderation by sex, race, and depressive symptoms. J Clin Endocrinol Metab. 2013;98:3470-81.

49. Selvin S. Statistical Analysis of Epidemiologic Data. 3rd edition. Oxford University Press, New York, NY 2004.

50. Hochberg Y, Tamhane AC. Multiple comparison procedures. New York: Wiley; 1987.

51. Beydoun MA, Fanelli Kuczmarski MT, Beydoun HA, Rostant OS, Evans MK, Zonderman AB. Associations of the ratios of $n-3$ to $n-6$ dietary fatty acids with longitudinal changes in depressive symptoms among US women. Am J Epidemiol. 2015;181: 691-705.

52. Copeland WE, Shanahan L, Worthman C, Angold A, Costello EJ. Cumulative depression episodes predict later $\mathrm{C}$-reactive protein levels: a prospective analysis. Biol Psychiatry. 2012;71:15-21.

53. Pasco JA, Nicholson GC, Williams LJ, Jacka FN, Henry MJ, Kotowicz MA, et al. Association of high-sensitivity C-reactive protein with de novo major depression. $\mathrm{Br} \mathrm{J}$ Psychiatry. 2010;197:372-7.

54. Gillespie CF, Garlow SJ, Binder EB, Schatzberg AF \& Nemeroff CB, editors. Textbook of psychopharmacology. America Psychiatric Publishing, Arlington, VA 2009.
55. Zhu CB, Lindler KM, Owens AW, Daws LC, Blakely RD, Hewlett WA. Interleukin-1 receptor activation by systemic lipopolysaccharide induces behavioral despair linked to MAPK regulation of CNS serotonin transporters. Neuropsychopharmacology. 2010;35:2510-20.

56. Neurauter G, Schrocksnadel K, Scholl-Burgi S, SpernerUnterweger B, Schubert C, Ledochowski M, et al. Chronic immune stimulation correlates with reduced phenylalanine turnover. Curr Drug Metab. 2008;9:622-7.

57. Felger JC, Li L, Marvar PJ, Woolwine BJ, Harrison DG, Raison CL, et al. Tyrosine metabolism during interferonalpha administration: association with fatigue and CSF dopamine concentrations. Brain Behav Immun. 2013;31: 153-60.

58. Maes M, Leonard BE, Myint AM, Kubera M, Verkerk R. The new '5-HT' hypothesis of depression: cell-mediated immune activation induces indoleamine 2,3-dioxygenase, which leads to lower plasma tryptophan and an increased synthesis of detrimental tryptophan catabolites (TRYCATs), both of which contribute to the onset of depression. Prog Neuropsychopharmacol Biol Psychiatry. 2011;35:702-21.

59. Raison CL, Dantzer R, Kelley KW, Lawson MA, Woolwine BJ, Vogt G, et al. CSF concentrations of brain tryptophan and kynurenines during immune stimulation with IFN-alpha: relationship to CNS immune responses and depression. Mol Psychiatry. 2010;15:393-403.

60. Steiner J, Walter M, Gos T, Guillemin GJ, Bernstein HG, Sarnyai $\mathrm{Z}$, et al. Severe depression is associated with increased microglial quinolinic acid in subregions of the anterior cingulate gyrus: evidence for an immune-modulated glutamatergic neurotransmission? J Neuroinflammation. 2011;8:94.

61. Tavares RG, Tasca CI, Santos CE, Alves LB, Porciuncula LO, Emanuelli T, et al. Quinolinic acid stimulates synaptosomal glutamate release and inhibits glutamate uptake into astrocytes. Neurochem Int. 2002;40:621-7.

62. Tilleux S, Hermans E. Neuroinflammation and regulation of glial glutamate uptake in neurological disorders. J Neurosci Res. 2007;85:2059-70.

63. Hardingham GE, Fukunaga Y, Bading H. Extrasynaptic NMDARs oppose synaptic NMDARs by triggering CREB shutoff and cell death pathways. Nat Neurosci. 2002;5:405-14.

64. Koo JW, Russo SJ, Ferguson D, Nestler EJ, Duman RS. Nuclear factor-kappaB is a critical mediator of stress-impaired neurogenesis and depressive behavior. Proc Natl Acad Sci USA. 2010;107:2669-74.

65. Goshen I, Kreisel T, Ben-Menachem-Zidon O, Licht T, Weidenfeld J, Ben-Hur T, et al. Brain interleukin-1 mediates chronic stress-induced depression in mice via adrenocortical activation and hippocampal neurogenesis suppression. Mol Psychiatry. 2008;13:717-28.

66. Haroon E, Woolwine BJ, Chen X, Pace TW, Parekh S, Spivey JR, et al. IFN-alpha-induced cortical and subcortical glutamate changes assessed by magnetic resonance spectroscopy. Neuropsychopharmacology. 2014;39:1777-85.

67. Haroon E, Fleischer CC, Felger JC, Chen X, Woolwine BJ, Patel $\mathrm{T}$, et al. Conceptual convergence: increased inflammation is associated with increased basal ganglia glutamate in patients with major depression. Mol Psychiatry. 2016;21:1351-7. 